\title{
Adult-Type Metachromatic Leukodystrophy Mimicking Multiple Sclerosis
}

\author{
Saber Chebel, Ilhem Barboura, Amel Boughammoura-Bouatay, Manel Ammar, Salima Ferchichi, \\ Abdelhedi Miled, Mahbouba Frih-Ayed
}

Can. J. Neurol. Sci. 2009; 36: 521-523

Metachromatic leukodystrophy (MLD) is an autosomal recessive lysosomal storage disease caused by a deficiency of arylsulphatase A (ASA). It is characterized by accumulation of sulphatide in the white matter in the central nervous system and peripheral nerves ${ }^{1}$.

The disease is divided in four subtypes, according to onset, severity and progression of the disease: late infantile (before age 4), early juvenile (age 4 to 6), late juvenile (age 6 to 16) and adult-type (after age 16) ${ }^{1}$. The disorder has different clinical presentations depending on the age at onset. Most patients with adult-type MLD present psychiatric symptoms and a schizophrenia-like condition ${ }^{2,3}$. More recent reports indicate that dementia together with pure neurological symptoms are also prominent ${ }^{3-5}$. Before examination by magnetic resonance imaging (MRI) or ASA activity dosage, the patients are often misdiagnosed as having schizophrenia, affective disorder, or personality disorder. We report a patient with an adult-type MLD, in which the clinical course and neuroimaging results could suggest the diagnosis of multiple sclerosis (MS).

\section{Case Report}

A 44-year-old woman presented a history of primary sterility. There were no family history of neurological or mental disease, nor had she had any previous episodes of neurotrauma or infection. The patient worked as a medical secretary. She was initially seen at the age of 18 years because of two episodes of generalized tonic and clonic seizures treated with Phenobarbital and patient was seizures-free since this date. In December 2003, the patient gradually developed a lack of interest, social withdrawal, blunted affect and she could not take care of her home and work. She showed signs of inappropriate giggling. In May 2005, when she first visited our department she became untidy and disinhibited. She lost depth of feeling and had no insight into her illness. She was less worried and had the feeling of euphoria. She had difficulty with abstract thinking and problem solving. At any time of the disease evolution, there was no hallucination or paranoid delusion and neither had she had any episodes of visual, or sensory nor motor disturbances. Neurological examination showed pyramidal features in all limbs without paresis (she had brisk reflexes in four limbs with bilateral Babinski sign). Cranial nerves examination, cerebellar, sensory, and gallbladder functions were all normal. Ophthalmologic examination and ocular funds were also normal. On the mini mental state examination, she scored 13 (perfect score: 30). Memory disturbance, disorientation, difficultymaintaining attention, calculating disturbance and disturbed word fluency were obvious. Neuropsychological cognitive testing including short cognitive evaluation battery (B2C), Rey's complex figure and Raven matrix showed a dementia with significant memory impairments, including the episodic, semantic and working memories. An involvement of the visuospatial and instrumentals functions, attention, problem solving, and amnesic language were also recorded. Her social competence and level of function had declined. Her psychomotor speed was decreased during more complex tasks, such as the Rey's complex figure. Memory performance for both visual and auditory presented material was also decreased. She was also anxious and emotional labile. No aphasia, apraxia or agnosia were noted. A brain magnetic resonance imaging MRI showed confluent white matter $\mathrm{T} 2$ and fluid attenuated inversion recovery (FLAIR) hyper intensities in the periventricular regions and corpus callosum (Figures $1 \mathrm{~A}$ and B) associated to a diffuse cerebral atrophy. Spinal cord and angiography MRI were normal. Routine clinical chemistry tests were all normal, including sedimentation rate, blood cell counts, C reactive protein, glycaemia, serum protein electrophoresis, haemostasis, phosphocalcic, lipid, thyroid, liver and kidney tests. No serum antibodies were detected against Borrelia, syphilis, human immunodeficiency virus (HIV), hepatitis B and C or Whipple disease. Anti-nuclear, anti-DNA, anti-SSA, anti-SSB and antiphospholipids antibodies were normal. The rheumatoid factor and cryoglobulineamia were not present. Organic and amines acids chromatography and serum levels of lactates, B12 vitamin and folates were normal. In the spinal fluid there was no pleocytosis, the spinal glucose value was $2.9 \mathrm{mmol} / \mathrm{l}$ (normal range (NR): $2.5-4.5$ ), and the spinal protein level was mildly increased $0.64 \mathrm{~g} / \mathrm{l}$ (NR: $0.15-0.50)$. The IgG index was not elevated, and no oligoclonal bands or abnormal cells (neoplastic or Periodic Acid Shiff-positive) were detected. Abdominal ultrasonography and upper digestive tract endoscopy were normal. No abnormalities were recorded on jejunal, axillary or salivary glands biopsies. Electroneuromyography, muscle biopsy and multimodal evoked potentials (sensory, brainstem auditory

\footnotetext{
From the Department of Neurology (SC, ABB, MA, MFA), University Hospital of Monastir, Monastir; Department of Biochemistry (IB, SF, AM), Farhat Hached University Hospital, Sousse, Tunisia.

Received October 7, 2008. Final Revisions Submitted February 4, 2009. Correspondence to: Saber Chebel, Department of Neurology, University Hospital of Monastir, Monastir 5000, Tunisia.
} 

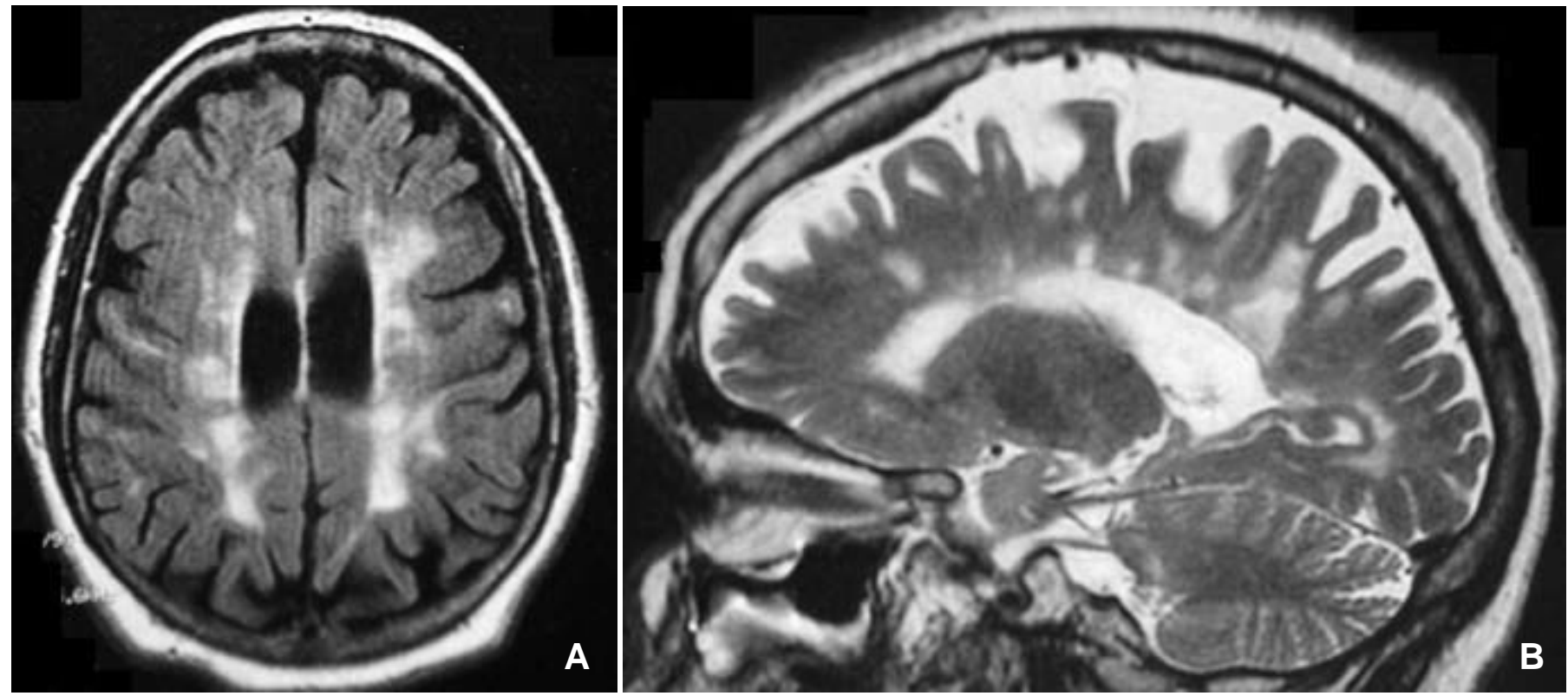

Figure 1: A) Axial brain MRI fluid-attenuated inversion recovery images showing confluent periventricular white matter hyper intensities. B) Sagittal brain MRI T2-weighted images showing corpus callosum involvement.

and visual) were normal. In two separate samples, ASA activity in peripheral blood leucocytes measured at $0^{\circ} \mathrm{C}$ and $37^{\circ} \mathrm{C}$ was decreased, with respective values of $1.3 \mathrm{nmol} / \mathrm{h} / \mathrm{mg}$ protein (NR: $3-9$ ) and $7.15 \mathrm{nmol} / \mathrm{h} / \mathrm{mg}$ protein (NR: 12-27). Thin-layer chromatography of urinary lipid extracts (qualitative essay) revealed high levels of sulfatide (3-sulfogalagtosylceramide) in urine. The ASA gene study revealed the, I179S, missense mutation.

In June 2007, she was admitted in the intensive care unit because of febrile coma with acute respiratory distress and status epilepticus. On neurological examination there were no signs of peripheral neuropathy. The brain MRI showed characteristic lesions of MLD with extensive demyelination, especially around the ventricles, and diffuse cerebral atrophy, without signs of encephalitis (Figure 2). The spinal fluid analysis showed no abnormalities, except a slight increased protein's level of 0.50 $\mathrm{g} / \mathrm{l}$. The patient died two weeks after admission because of uncontrolled and severe septicaemia.

\section{Discussion}

The present case of a young adult patient showed gradual obvious dementing symptoms such as memory disturbance and disorientation. In the absence of aphasia, agnosia or apraxia, the character of dementia in our case is different from that of Alzheimer's disease, which affects the cortical gray matter. The clinical features together with character change and intellectual decline are thought to be a manifestation of a white matter dementia seen in various diseases involving the cerebral white matter such as genetic leukodystrophies, Binswanger type of cerebral vascular dementia, metabolic (e.g. deficiency of vitamin B12 and folates) or infectious diseases (e.g. HIV infection, and progressive multifocal leukoencephalopathy), inflammatory diseases (eg. lupus cerebritis) and $\mathrm{MS}^{6}$. In the present case, the cognitive deficits encompassed several cognitive domains including memory, visuo-spatial, and executive functions indicating widespread organic lesion(s), and based on the initial brain MRI, showing a confluent white matter T2 and FLAIR hyperintensities in the periventricular regions and the corpus callosum indicating demyelination. At the first time, and because of the normality of all inflammatory and infectious diseases diagnosis tests, a primary progressive MS (form characterized by a progressive evolution of clinical symptoms without relapses since the disease onset) was considered the most likely diagnosis. In this fact, cognitive impairment in MS is well recognized and appears to be common even in the early stages $^{7-10}$. However, pure cognitive or neuropsychiatric forms of MS (conditions used to describe patients with prominent or isolated neurobehavioral symptoms and meeting MS criteria's) are less frequent and less studied, with generally, a limited reported number of patients ${ }^{7}$. The clinical features of these forms are, a mean age of diagnosis of 45 years, the primary progressive form was the most frequent, a mild expanded disability status scale (2 to 3 ) and a mean duration of the disease evolution of 2.5 to 7.5 years. The cognitive impairment affects mainly attention, memory, inhibition, information processing speed, and executive functions. Magnetic resonance imaging (MRI) lesions are similar to the "classic" disease forms ${ }^{10}$. All these conditions were present in our patient, but there were some major conditions inconsistent with the diagnosis of MS: First, at the age of 18 the patient developed generalized epileptic seizures, which are an exceptional condition in MS. Second, during all the disease evolution the patient did not complain of any sensory or visual symptoms. Third, the brain MRI lesions were consistent with MS, however, the spinal cord was not involved, and finally multimodal evoked potentials were normal. Because of all these reasons, the diagnosis of other white matter disease especially of metabolic origin such as MLD or mitochondrial disease was considered and a new screening tests including ASA activity 


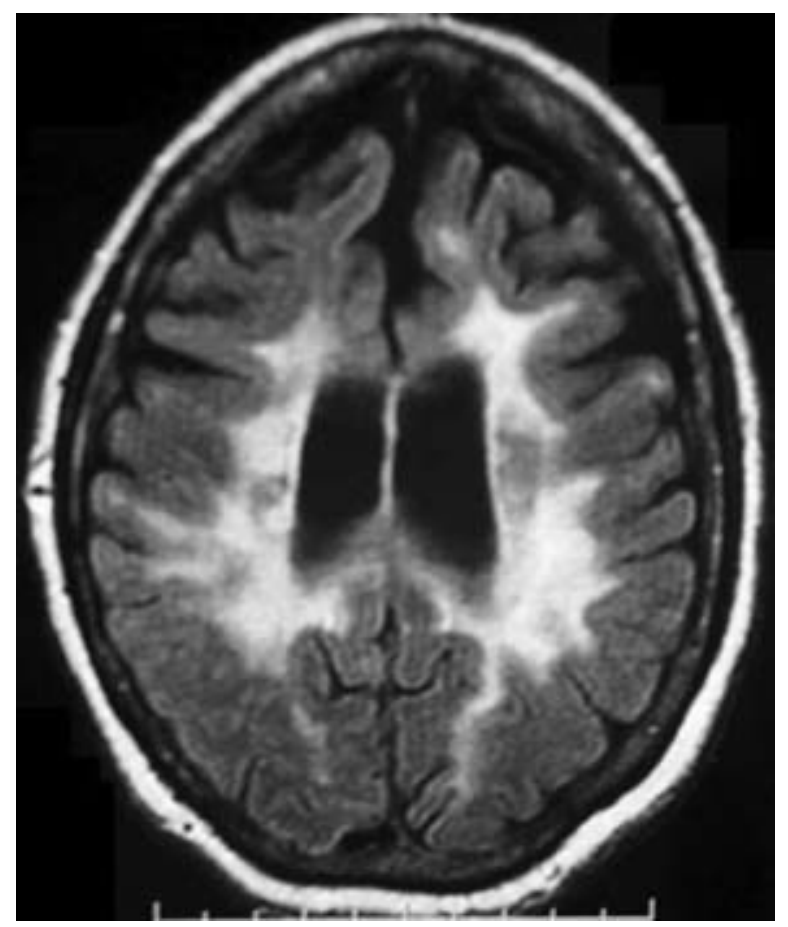

Figure 2: Axial brain MRI fluid-attenuated inversion recovery images two years later, showing diffuse, bilateral and symmetrical areas of hyperintensities in the periventricular and sub cortical white matter.

dosage were performed and concluded to the diagnosis of MLD of the adult-type according to the age of onset ${ }^{1}$. A large number of MLD patients with psychosis and dementia have been reported, however, there have been few case reports, confirmed by gene analysis, with detailed descriptions of the psychiatric features ${ }^{2}$. Most MLD cases have an infantile or a juvenile onset. However, a few patients with adult onset MLD, usually in the second or third decades of life, have been reported. In more recent papers ${ }^{3-5}$, two clinical and genetic distinct forms of adulttype MLD were reported: Motor forms involved the major adult ASA mutation P426L in a homozygote form, in contrast to the psychocognitive forms which involved as a compound heterozygote a specific I179S mutation, like seen in our patient.

The reported case had some particularities: First, generalized epileptic seizures were inaugural of the disease, and the patient developed a status epilepticus in the late stage. Indeed epileptic seizures are observed at a relatively late stage of MLD in $25 \%$ of patients with late infantile form and in 50 to $60 \%$ of patients with juvenile form. Late onset cases of MLD are rare and a small number of patients with late juvenile or adult onset MLD have been reported in the literature ${ }^{4,11} \cdot 2.2 \%$ of them had seizures at onset, while $22.7 \%$ developed seizures later in the course of the disease. There were no seizures-type descriptions in the adult form of the disease. Further recurrent seizures and status epilepticus have been reported only in some cases of infantile and juvenile MLD, but not in adult forms ${ }^{11,12}$. Second, in the initial brain MRI hyperintensities were MS-like lesions. Typically, MRI evidenced in MLD a diffuse bilateral demyelination, often symmetrical, and respecting $U$ fibres.
These white matter changes were mainly in frontal regions and associated to cortical and subcortical atrophy ${ }^{3,4}$. Finally, we have reported the case of an adult-type MLD with no clinical or neurophysiologic evidence of peripheral neuropathy during all the disease evolution with, I179S ASA gene mutation, which has been tentatively associated to a late onset MLD, particularly when psychiatric symptoms are prominent. At our knowledge, in the rare earlier described cases of MLD without peripheral neuropathy, only Gallo et $\mathrm{al}^{13}$, report a compound heterozygote for two previously unreported missense mutations in the ASA gene, G293D in exon 5, and C489G mutation in exon 8.

The clinical presentation of our patient was particular because of the absence of a detectable peripheral nervous system involvement, and white matter lesions on the initial brain MRI. These clinical and especially neuroimaging features could suggest a cognitive presentation of MS of the progressive form. However, the presentation with neurobehavioral symptoms was otherwise suggestive of another white matter involving disease, like adult-onset MLD.

We recommend in all young adults with neurobehavioral symptoms and MS-like white matter hyper intensities, a full sequential diagnostic search including the screening of acquired, and genetic leukodystrophies especially MLD.

\section{REFERENCES}

1. Von Figura K, Gieselmann V, Jaeken J. Metachromatic leukodystrophy. In: Scriver CR, Beaudet AL, Valle D, Sly WS, editors. The metabolic basis of inherited disease. 8th ed. New York: McGraw-Hill; 2001: p. 3695-724.

2. Johannsen P, Ehlers L, Hansen HJ. Dementia with impaired temporal glucose metabolism in late-onset metachromatic leukodystrophy. Dement Geriatr Cogn Disord. 2001; 12: 85-8.

3. Baumann N, Turpin JC, Lefevre M, Colsch B. Motor and psychocognitive clinical types in adult metachromatic leukodystrophy: genotype/phenotype relationships? J Physiol. 2002; 96: 301-6.

4. Rauschka H, Colsch B, Baumann N, Wevers R, Schmidbauer M, Krammer M, et al. Late-onset metachromatic leukodystrophy. Genotype strongly influences phenotype. Neurology. 2006; 67: 859-63.

5. Colsch B, Afonso C, Turpin JC, Portoukalian J, Tabet JC, Baumann N. Sulfogalactosylceramides in motor and psycho-cognitive adult metachromatic leukodystrophy: relations between clinical, biochemical analysis and molecular aspects. Biochim Biophys Acta. 2008; 1780: 434-40.

6. Pantoni L, Poggesi A, Inzitari D. The relation between white-matter lesions and cognition. Curr Opin Neurol. 2007; 20: 390-7.

7. Zarei M, Chandran S, Compston A, Hodges J. Cognitive presentation of multiple sclerosis: evidence for a cortical variant. J Neurol Neurosurg Psychiatry. 2003; 74: 872-7.

8. Feinstein A. The neuropsychiatry of multiple sclerosis. Can J Psychiatry. 2004; 49: 157-63.

9. Deloire MS, Salort E, Bonnet M, Arimone Y, Boudineau M, Amieva $\mathrm{H}$, et al. Cognitive impairment as marker of diffuse brain abnormalities in early relapsing remitting multiple sclerosis. J Neurol Neurosurg Psychiatry. 2005;76:519-26.

10. Brochet B, Bonnet M, Deloire M, Hamel D, Salort-Campana E. Les troubles cognitifs au cours de la sclérose en plaques. Rev Neurol. 2007; 163: 697-702.

11. Bostantjopoulou S, Katsarou Z, Michelakaki H, Kazis A. Seizures as a presenting feature of late onset metachromatic leukodystrophy. Acta Neurol Scand. 2000; 102: 192-5.

12. Balslev T, Cortez MA, Blaser SI, Haslam RH. Recurrent seizures in metachromatic leukodystrophy. Pediatr Neurol. 1997; 17: 150-4.

13. Gallo S, Randi D, Bertelli M, Salviati A, Pandolfo M. Late onset MLD with normal nerve conduction associated with two novel missense mutations in the ASA gene. J Neurol Neurosurg Psychiatry. 2004; 75: 655-7. 